\title{
Video Article \\ Atmospheric-pressure Molecular Imaging of Biological Tissues and Biofilms by LAESI Mass Spectrometry
}

\author{
Peter Nemes ${ }^{1}$, Akos Vertes ${ }^{1}$ \\ ${ }^{1}$ Department of Chemistry, George Washington University \\ Correspondence to: Akos Vertes at vertes@gwu.edu \\ URL: https://www.jove.com/video/2097 \\ DOI: doi:10.3791/2097
}

Keywords: Molecular Biology, Issue 43, imaging mass spectrometry, ambient mass spectrometry, direct analysis, tissue, biofilm

Date Published: 9/3/2010

Citation: Nemes, P., Vertes, A. Atmospheric-pressure Molecular Imaging of Biological Tissues and Biofilms by LAESI Mass Spectrometry. J. Vis. Exp. (43), e2097, doi:10.3791/2097 (2010).

\section{Abstract}

Ambient ionization methods in mass spectrometry allow analytical investigations to be performed directly on a tissue or biofilm under nativelike experimental conditions. Laser ablation electrospray ionization (LAESI) is one such development and is particularly well-suited for the investigation of water-containing specimens. LAESI utilizes a mid-infrared laser beam ( $2.94 \mu \mathrm{m}$ wavelength) to excite the water molecules of the sample. When the ablation fluence threshold is exceeded, the sample material is expelled in the form of particulate matter and these projectiles travel to tens of millimeters above the sample surface. In LAESI, this ablation plume is intercepted by highly charged droplets to capture a fraction of the ejected sample material and convert its chemical constituents into gas-phase ions. A mass spectrometer equipped with an atmospheric-pressure ion source interface is employed to analyze and record the composition of the released ions originating from the probed area (pixel) of the sample. A systematic interrogation over an array of pixels opens a way for molecular imaging in the microprobe analysis mode. A unique aspect of LAESI mass spectrometric imaging is depth profiling that, in combination with lateral imaging, enables threedimensional (3D) molecular imaging. With current lateral and depth resolutions of $\sim 100 \mu \mathrm{m}$ and $\sim 40 \mu \mathrm{m}$, respectively, LAESI mass spectrometric imaging helps to explore the molecular structure of biological tissues. Herein, we review the major elements of a LAESI system and provide guidelines for a successful imaging experiment.

\section{Video Link}

The video component of this article can be found at https://www.jove.com/video/2097/

\section{Protocol}

The following protocol describes the major steps of the laser ablation electrospray ionization (LAESI) experiment and provides representative examples for lateral and three-dimensional (3D) imaging for animal and plant tissue samples. Additional experimental and technical details can be obtained from elsewhere.

\section{Tissue Preparation and Mounting}

1. If sectioning is needed, use a cryomicrotome to section the tissue into $10-100 \mu \mathrm{m}$ thick slices at -10 to $-20^{\circ} \mathrm{C}$ unless otherwise recommended for a particular tissue type.

2. Mount sections onto a flat surface (e.g., chemically pre-cleaned glass slide) directly without chemical modifiers. For sectioned tissues, thawmount the sections and secure the sample holder to a Peltier-cooling stage immediately upon thaw-mounting to keep the tissue frozen at all times during analysis. This step is needed to minimize/prevent molecular migration in the section.

3. If needed, use a heat sink equipped with a low-power fan to facilitate heat removal from the Peltier-stage to maintain the tissue frozen.

4. In a humid environment over an extended period of time (1-2 hours), inspect for condensation of water or ice on the tissue surface. Water condensation on the tissue detrimentally affects the imaging performance in LAESI experiments. ${ }^{1}$

5. If needed, use a room dehumidifier or place the cooled sample in an environmental chamber filled with an inert gas (e.g., dry nitrogen gas) to prevent condensation. ${ }^{1}$

\section{Optimization of the LAESI Ion Source}

The LAESI ion source consists of a mid-infrared laser, a series of optical elements for light steering and focusing as well as additional sample holders, cooling components, translation stages, and a solvent delivery system. Figure 1 demonstrates the typical arrangement of these elements with respect to the entrance of the atmospheric-pressure ion source of a mass spectrometer.

1. As illustrated in Figure 1, position the sample $15-20 \mathrm{~mm}$ below the orifice of the mass spectrometer sampling cone ( $\mathrm{d}_{\mathrm{OR}-\mathrm{FP}}$ ).

2. Operate a mid-IR laser at $2.94 \mu \mathrm{m}$ wavelength and $10 \mathrm{~Hz}$ repetition rate. Attenuate the laser output to $\sim 100 \mu \mathrm{J} /$ pulse energy. 
3. Use a combination of gold mirrors and a focusing lens transparent at the laser wavelength (e.g., a plano-convex $\mathrm{CaF}_{2}$ or $\mathrm{ZnSe}$ lens) to couple the laser pulse energy into the sample at normal incidence (see right incidence angle with respect to sample surface in Figure 1).

4. Position the mid-infrared beam axis $5-8 \mathrm{~mm}$ in front of the orifice of the mass spectrometer sampling cone.

5. Adjust the focusing lens position and the pulse energy of the laser beam to achieve tissue removal in the focal spot. The dimensions of the ablated volume determine the pixel (or voxel in three-dimensional imaging) size for the imaging application.

6. Position a nanospray emitter in line with the inlet axis of the mass spectrometer and at an orifice-to-emitter tip distance of $\sim 10 \mathrm{~mm}$ (see Figure 1).

7. For the electrospray, prepare $50 \%$ methanol solution with $0.1 \%$ acetic acid or $0.1 \%$ ammonium acetate additive for positive or negative ion mode, respectively. Depending on the sample, other organic solvents, such as acetonitrile, isopropanol, etc., may replace methanol at concentrations appropriate for the analytical task. The stability of the electrospray is crucial for successful imaging. Depending on the solvent selection, the flow rate and the spraying voltage need to be adjusted to achieve stable spray.

8. For reactive $\mathrm{LAESI}^{6}$ in imaging applications, the electrosprayed solution may contain reactants.

9. Use a syringe pump to deliver the electrospray solution through the electrospray emitter at a flow rate of $\sim 300 \mathrm{~nL} / \mathrm{min}$.

10. If the mass spectrometer orifice is kept at a low voltage $(<500 \mathrm{~V}$ measured against ground), generate electrospray by applying high voltage directly to the electrospray emitter (e.g., $3000 \mathrm{~V}$ ) or through a metal union. Otherwise, ground directly the electrospray emitter or through metal union to establish electrospray.

11. Operate the electrospray source in cone-jet spraying mode for most efficient ion generation by LAESI. For the effect of operating variables on the spraying modes and their effect on the mass spectra, see discussions elsewhere., ${ }^{5,7}$

12. Carefully adjust the relative distances of the LAESI setup to optimize for LAESI ion yield while keeping the laser beam, the emitter, and the orifice axes in the same plane. Please find detailed instructions elsewhere. ${ }^{5}$

13. With an optical microscope, determine the lateral dimensions of the ablation crater on the sample.

14. For three-dimensional LAESI imaging experiments, perform ablation with individual pulses and determine the depth of a voxel using, for example, the z-stack mode in optical microscopy. ${ }^{3}$

\section{Molecular Imaging and Data Analysis}

In the imaging experiment, the tissue sample is moved in the focal plane of the laser in $\mathrm{X}$ and $\mathrm{Y}$ directions with step sizes larger than or equal to the dimensions of the ablation spot. The spatial resolution is limited by the focusing of the incident laser beam.

1. Select the area of interest on the sample surface and obtain the $(X, Y)$ coordinates of the corresponding boundaries.

2. Chose a gridding algorithm (e.g., adaptive grid, selected region imaging, rectangular grid, spiral pattern, $Z$ scanning, etc.) with which to raster the sample surface with selected dwell time at each pixel over the area to be imaged.

3. Use a three-axis translation stage and software that is capable of rastering the sample according to the predetermined grid.

4. Calculate the total time required for imaging.

5. Disable the data acquisition time limit of the mass spectrometer. If this is not possible, set the data acquisition time limit to the calculated imaging time value.

6. Start the mid-infrared laser source at a repetition rate proper to produce sufficient signal-to-noise ratio in the mass spectrum within the dwell time at each pixel to perform a LAESI lateral imaging experiment. For 3D molecular imaging, use a spectrum acquisition rate higher than the laser source repetition rate to successfully mass-analyze the ions generated within a single laser pulse. Wait for the START signal to initiate the ablation sequence.

7. Turn on the electrospray source. Make sure that there is enough solution for the full time required for imaging.

8. Simultaneously START the acquisition of mass spectra, the mid-IR laser ablation, and the surface scanning.

9. When the imaging run has finished, STOP the surface scanning, the mid-IR lasing, and the data acquisition.

10. Disable the laser source.

11. Turn off the high voltage.

12. Switch off the syringe pump.

13. Set the mass spectrometer to STANDBY mode.

14. Turn off the Peltier-cooling electronics.

15. Close inert gas flow if used.

16. Use a software to correlate the absolute coordinates of the pixels in lateral imaging or the voxels in $3 \mathrm{D}$ analysis with the corresponding spectra.

17. Plot the ion intensity signal for a selected $\mathrm{m} / \mathrm{z}$ value against the absolute coordinates of analysis to obtain lateral and 3D molecular images.

\section{Representative Results}

Figure 2 gives representative results for some major tissue types and imaging modalities. Panel A depicts a case for an animal tissue section that has been frozen during the experiment to prevent dehydration. ${ }^{1}$ In addition, the sample was located in a dry nitrogen gas environment to avoid ambient water vapors from condensing on the sample surface. A $100-\mu \mathrm{m}$-thick coronal section of a rat brain (Rattus norvegicus) was laterally imaged with LAESI. The anatomical regions of the brain (see optical image in panel A) show good correlation with the molecular image obtained for the plasmalogens $\mathrm{PC}(\mathrm{O}-33: 3)$ and/or $\mathrm{PE}(\mathrm{O}-36: 3)$ with $\mathrm{m} / \mathrm{z} 728.559$.

Panel B shows the 3D LAESI imaging of a Zebra plant (Aphelandra squarrosa) leaf tissue. Because leaves possess a natural defense mechanism against dehydration, the sample could be interrogated in the ambient environment. ${ }^{3}$ The obtained 3D molecular images revealed a variety of distribution patterns for primary and secondary metabolites. Among others, acacetin with $\mathrm{m} / \mathrm{z} 285.076$ was detected at higher ion counts in the yellow sectors of the second and third layers from the top with a homogeneous distribution in the others. This distribution agreed with the pattern of the variegation seen in the optical image. 


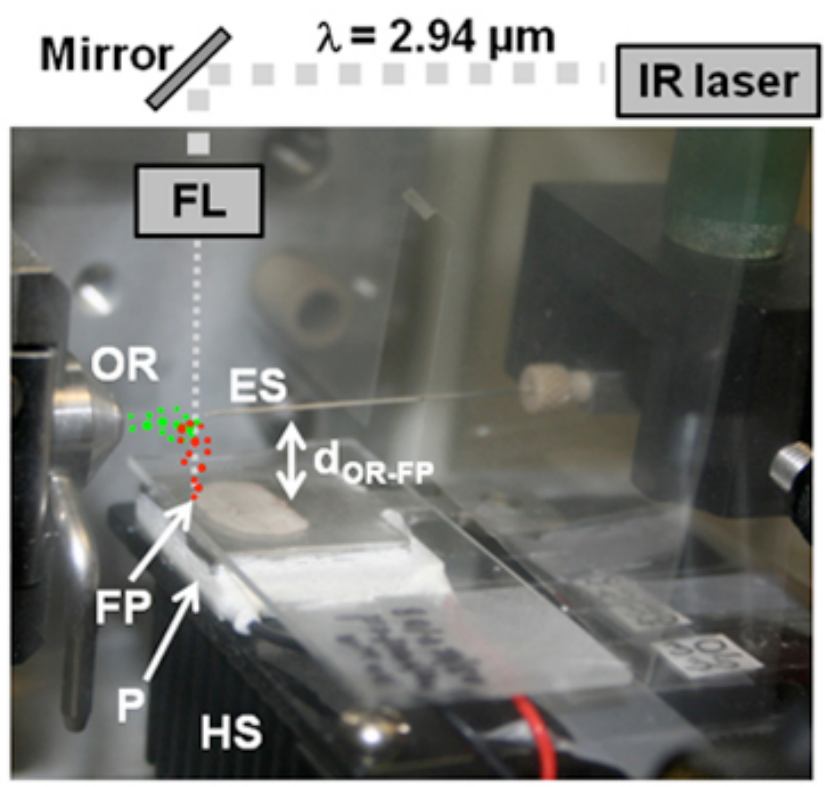

Figure 1. Schematic of the LAESI system (ES, electrospray emitter tip; OR, orifice of mass spectrometer sampling cone; FL, focusing lens; FP, focal point; P, Peltier cooling stage; HS, heat sink). A portion of the particulate matter expelled during mid-IR ablation (red dots) coalesces with the electrospray to yield charged droplets seeded with molecules and ions of the sample (green dots). The ions released from these droplets are analyzed and recorded by the mass spectrometer.
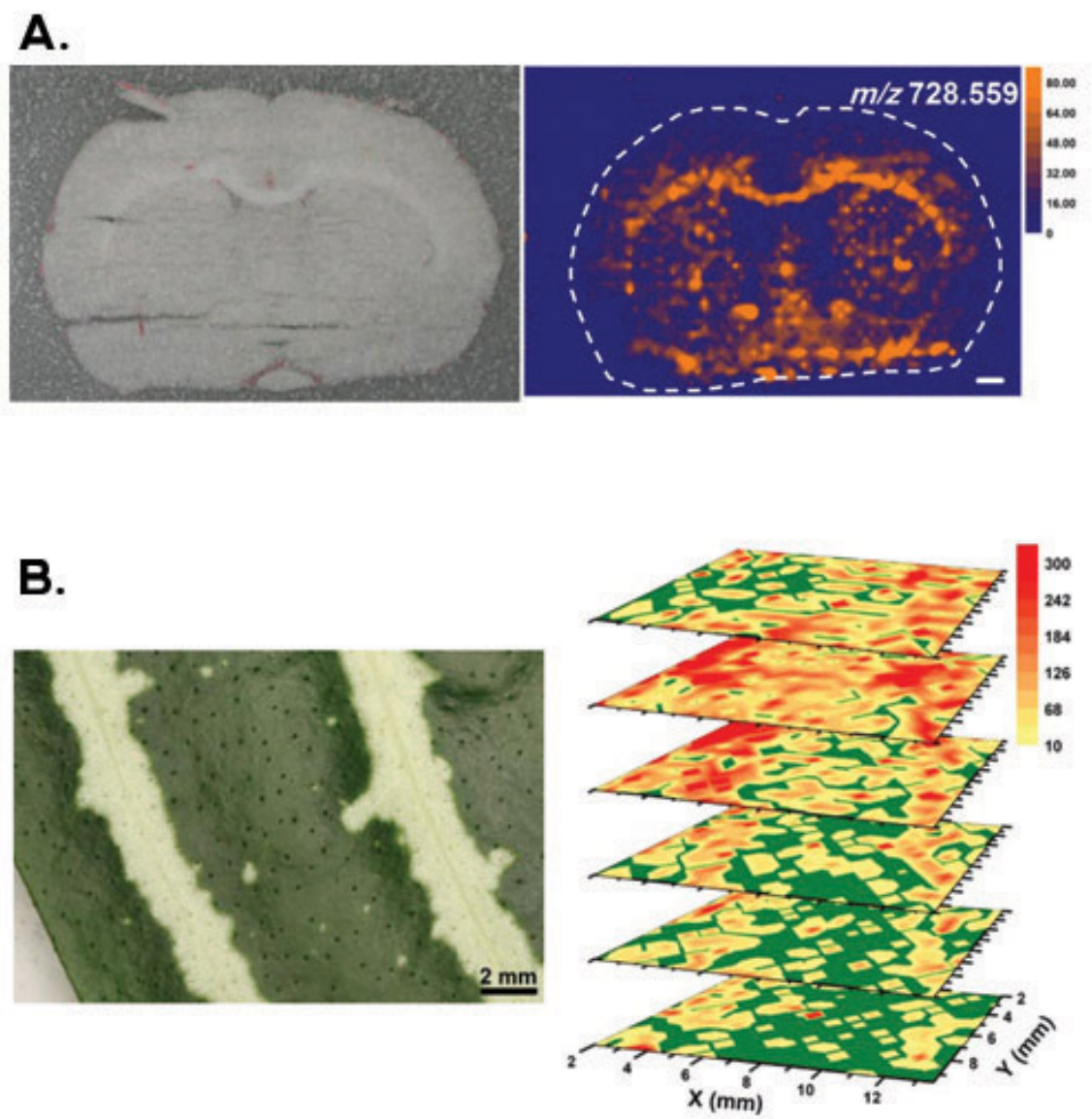

Figure 2. Representative results for lateral and 3D imaging with LAESI mass spectrometry. (A) The top panel depicts the optical image of a rat brain (Rattus norvegicus) coronal section and the molecular image obtained for the plasmalogens $\mathrm{PC}(\mathrm{O}-33: 3)$ and/or $\mathrm{PE}(\mathrm{O}-36: 3)$ with $m /$ z 728.559. The white scale bar corresponds to $1 \mathrm{~mm}$. Adapted with permission from (Reference ${ }^{1}$ ). Copyright 2010 American Chemical Society. (B) The bottom panel shows the 3D imaging of a leaf from a variegated Zebra plant (Aphelandra squarrosa). Acacetin with $\mathrm{m} / \mathrm{z} 285.076$ was 
detected at higher ion counts in the yellow sectors of the second and third layers from the top with a homogeneous distribution in the others. Reprinted with permission from (Reference ${ }^{3}$ ). Copyright 2009 American Chemical Society.

\section{Discussion}

Different tissue types exhibit varying water content and tensile strength, which, in turn, can affect the ablation characteristics of the samples. ${ }^{9}$ To mitigate these effects, it is desired that the laser fluence, sample handling, and analysis protocols be revised when changing between major tissue types.

For single cell or higher resolution investigations, the mid-infrared light can be coupled into a sharpened optical fiber instead of a focusing lens. ${ }^{10}$ By positioning the fiber tip within close proximity of the selected cells in a tissue, LAESI analysis can be performed on a single-cell level.

As a label-free ion source for ambient ionization mass spectrometry, ${ }^{11}$ LAESI has shown great potential for the investigation of biochemical processes in tissues. With the added benefits of direct analysis, lateral and 3D imaging, LAESI is an emerging bioanalytical tool for profiling as well as imaging applications.

\section{Disclosures}

No conflicts of interest declared.

\section{Acknowledgements}

The authors are grateful for the financial support of this work by the U.S. National Science Foundation under Grant No. 0719232 , by the U.S. Department of Energy (DEFG02-01ER15129), and by Protea Biosciences, Inc. (Morgantown, WV). The authors would also like to thank Jessica A. Stolee for her help during the videotaping of the protocol.

\section{References}

1. Nemes, P., Woods, A. S. \& Vertes, A. Simultaneous Imaging of Small Metabolites and Lipids in Rat Brain Tissues at Atmospheric-Pressure by Laser Ablation Electrospray Ionization Mass Spectrometry. Anal Chem 82, 982-988 (2010).

2. Nemes, P. \& Vertes, A. Laser ablation electrospray ionization for atmospheric-pressure, in vivo, and imaging mass spectrometry. Anal Chem 79, 8098-8106 (2007).

3. Nemes, P., Barton, A. A. \& Vertes, A. Three-dimensional imaging of metabolites in tissues under ambient conditions by laser ablation electrospray ionization mass spectrometry. Anal Chem 81, 6668-6675 (2009).

4. Nemes, P., Barton, A. A., Li, Y. \& Vertes, A. Ambient molecular imaging and depth profiling of live tissue by infrared laser ablation electrospray ionization mass spectrometry. Anal Chem 80, 4575-4582 (2008).

5. Nemes, P. \& Vertes, A. Laser ablation electrospray ionization for atmospheric-pressure molecular imaging mass spectrometry. Mass Spectrometry Imaging, in Methods in Molecular Biology, Vol. 656, (eds) S. S. Rubakhin \& J. V. Sweedler, Springer in press (2010).

6. Shrestha, B. et al. Direct analysis of lipids and small metabolites in mouse brain tissue by AP IR-MALDI and reactive LAESI mass spectrometry. Analyst 135, 751-758 (2010).

7. Nemes, P., Marginean, I. \& Vertes, A. Spraying mode effect on droplet formation and ion chemistry in electrosprays. Anal Chem 79 , 3105-3116 (2007).

8. Nemes, P., Goyal, S. \& Vertes, A. Conformational and noncovalent complexation changes in proteins during electrospray ionization. Anal Chem 80, 387-395 (2008).

9. Vertes, A. et al. Molecular imaging by Mid-IR laser ablation mass spectrometry. Appl Phys A-Mater Sci Process 93, 885-891 (2008).

10. Shrestha, B. \& Vertes, A. In situ metabolic profiling of single cells by laser ablation electrospray ionization mass spectrometry. Anal Chem 81 , 8265-8271 (2009).

11. Cooks, R. G., Ouyang, Z., Takats, Z. \& Wiseman, J. M. Ambient mass spectrometry. Science 311, 1566-1570 (2006). 